\title{
On Various $g$-Topology in Statistical Metric Spaces
}

\author{
V. Renukadevi ${ }^{1 *}$ and S. Vadakasi ${ }^{2}$ \\ ${ }^{1}$ Centre for Research and Post Graduate Studies in Mathematics, Ayya Nadar Janaki Ammal College(Autonomous), Sivakasi-626 124, Tamil \\ Nadu, India. \\ ${ }^{2}$ Ayya Nadar Janaki Ammal College(Autonomous), Sivakasi-626 124, Tamil Nadu, India. \\ * Corresponding author
}

\author{
Article Info \\ Keywords: SM space, type $V_{D}$, g-écart- \\ g-topology, R-g-topology. \\ 2010 AMS: 54A08, 54A10 \\ Received: 6 May 2019 \\ Accepted: 3 August 2019 \\ Available online: 30 September 2019
}

\begin{abstract}
The purpose of this paper is to analyze the significance of new $g$-topologies defined in statistical metric spaces and we prove various properties for the neighbourhoods defined by Thorp in statistical metric spaces. Also, we give a partial answer to the questions, namely "What are the necessary and sufficient conditions that the $g$-topology of typeV to be of type $V_{D}$ ?, the $g$-topology of type $V_{\alpha}$ to be the $g$-topology of $t y p e V_{D}$ ? and the $g$-topology of type $V_{\alpha}$ to be a topology?" raised by Thorp in 1962. Finally, we discuss the relations between $\lambda_{\Omega}$-open sets in generalized metric spaces and various $g$-topology neighbourhoods defined in statistical metric spaces. Also, we prove weakly complete metric space is equivalent to a complete metric space if $\Omega$ satisfies the $\mathscr{V}$-property.
\end{abstract}

\section{Introduction}

Fréchet introduced the notion of an abstract metric space in the year 1906 [1] from which the concept of "distance" appears. The notion of distance is defined in terms of functions, points and sets. Indeed, in many situations, it is appropriate to look upon the distance concept as a statistical rather than a determinate one. More precisely, instead of associating a number to the distance d(p, q) with every pair of points p, q, one should associate a distribution function $F_{p q}$ and for any positive number $x$, interpret $F_{p q}(x)$ as the probability that the distance from $p$ to $q$ be less than $x$.

Using this idea, Menger [3] defined a statistical metric space using the probability function in the year 1942. In 1943, shortly after the appearance of Menger's article, Wald [10] published an article in which he criticized Menger's generalized triangle inequality. In 1951, Menger [5] continued his study of statistical metric spaces and in [4], he studied the behaviour of probabilistic theory.

In 1960, Schweizer et. al gave some properties of neighbourhoods defined by Thorp [7]. Thorp introduced some $g$-topologies in a statistical metric space [9] and he studied the properties of $t$-function in [8]. Further, Thorp proved some results using $g$-topologies defined in a statistical metric space [9]. Finally, he raised some questions about the relationship between various $g$-topologies defined in [9].

A statistical metric space (SM space) [9] is an ordered pair $(S, F)$ where $S$ is a non-null set and $F$ is a mapping from $S \times S$ into the set of distribution functions (that is, real-valued functions of a real variable which are everywhere defined, non decreasing, left-continuous and have infimum 0 and supremum 1$)$.

The distribution function $F(p, q)$ associated with a pair of points $p$ and $q$ in $S$ is denoted by $F_{p q}$. Moreover, $F_{p q}(x)$ represents the probability that the "distance" between $p$ and $q$ is less than $x$.

The functions $F_{p q}$ are assumed to satisfy the following:

(SM-I) $F_{p q}(x)=1$ for all $x>0$ if and only if $p=q$.

(SM-II) $F_{p q}(0)=0$.

(SM-III) $F_{p q}=F_{q p}$.

(SM-IV) If $F_{p q}(x)=1$ and $F_{q r}(y)=1$, then $F_{p r}(x+y)=1$.

We often find it convenient to work with the tails of the distribution functions rather than with these distribution functions themselves. Then the tail [9] of $F_{p q}$, denoted by $G_{p q}$, is defined by $G_{p q}(x)=1-F_{p q}(x)$ for all $x \in \mathbb{R}$.

Let $(S, F)$ be a statistical metric space. Then the menger inequality is,

(SM-IVm) $F_{p r}(x+y) \geq T\left(F_{p q}(x), F_{q r}(y)\right)$ holds for all points $p, q, r \in S$ and for all numbers $x, y \geq 0$ where $T$ is a 2-place function on the 
unit square satisfying:

(T-I) $0 \leq T(a, b) \leq 1$ for all $a, b, c \in[0,1]$.

(T-II) $T(c, d) \geq T(a, b)$ if $c \geq a, d \geq b$ (monotonicity) for all $a, b, c, d \in[0,1]$.

(T-III) $T(a, b)=T(b, a)$ (commutativity) for all $a, b \in[0,1]$.

(T-IV) $T(1,1)=1$.

(T-V) $T(a, 1)>0$ for all $a>0$.

\section{Preliminaries}

In this section, we recall some basic definitions in [9] and give some examples for these definitions in a statistical metric space.

Let $(S, F)$ be a statistical metric space, $p \in S$ and $u, v$ be positive numbers. Then $N_{p}(u, v)=\left\{q \in S \mid F_{p q}(u)>1-v\right\}=\left\{q \in S \mid G_{p q}(u)<v\right\}$

[9] is called the $(u, v)$-sphere with center $p$.

The following Example 2.1 shows that the existence of $(u, v)$-sphere in a statistical metric space.

Example 2.1. Consider the SM space $(S, F)$ where $S$ denotes the possible outcomes of getting a tail when a coin is tossed once. Then $S=\{0,1\}$. Here $F_{p q}(u)$ is the probability that the "distance" between $p$ and $q$ is less than $u$ where $u>0$ and $p, q \in S$. Fix $p=0$. Then

$$
N_{p}(u, v)=\left\{\begin{array}{lll}
S & \text { if } & 0<u<1, v>1, \\
S & \text { if } & u>1, v>0, \\
\{0\} & \text { if } & 0<u<1,0<v<1, \\
S & \text { if } & u>1, v>1 .
\end{array} .\right.
$$

Fix $p=1$. Then

$$
N_{p}(u, v)=\left\{\begin{array}{lll}
S & \text { if } & 0<u<1, v>1, \\
S & \text { if } & u>1, v>0, \\
\{1\} & \text { if } & 0<u<1,0<v<1, \\
S & \text { if } & u>1, v>1 .
\end{array} .\right.
$$

For fixed positive numbers $u$ and $v$, define $U(u, v)[9]$ by $U(u, v)=\left\{(p, q) \in S \times S \mid G_{p q}(u)<v\right\}$.

Example 2.2. Consider the SM space $(S, F)$ where $S$ denotes the possible outcomes of rolling a dice. Then $S=\{1,2,3,4,5,6\}$ and the distribution function $F_{p q}(x)$ is the probability that the "distance" between $p$ and $q$ is less than $u$ where $u>0$ and $p, q \in S$. Then $U(u, v)=\{(p, q) \in S \times S: p=q\}$ for $0<u<1,0<v<1$. For $1<u<2,0<v<1, U(u, v)=\{(p, q) \in S \times S: d(p, q) \leq 1\}$. For $2<u<3,0<v<1, U(u, v)=\{(p, q) \in S \times S: d(p, q) \leq 2\}$. For $3<u<4,0<v<1, U(u, v)=\{(p, q) \in S \times S: d(p, q) \leq 3\}$. For $4<u<5,0<v<1, U(u, v)=\{(p, q) \in S \times S: d(p, q) \leq 4\}$. For $u>5,0<v<1, U(u, v)=\{(p, q) \in S \times S: d(p, q) \leq 5\}=S \times S$. Now $0<u<1, v>1$. Then $U(u, v)=S \times S$. Also, $U(u, v)=S \times S$, for $u>1, v>1$.

For any set $Z$ of ordered pairs of positive numbers, $\mathscr{N}(Z)=\left\{N_{p}(u, v) \mid(u, v) \in Z, p \in S\right\}$ and $\mathscr{U}(Z)=\{U(u, v) \mid(u, v) \in Z\}$.

A non-null collection $\left\{N_{p}\right\}$ of subsets $\mathscr{N}(Z)$ in a set $S$ associated with a point $p \in S$ is a family of neighbourhoods for $p$ if each $N_{p}$ contains $p$. Let the family of neighbourhoods be associated with each point $p$ of a set $S$. The set $S$ and the collection of neighbourhoods is called the $g$-topological space of typeV [9].

Using the following conditions, Thorp [9] introduced new $g$-topologies in a statistical metric space $(S, F)$. No. typeV.

$N_{1}$. For each point $p$ and each neighbourhood $U_{p}$ of $p$, there is a neighbourhood $W_{p}$ of $p$ such that for each point $q$ of $W_{p}$, there is a neighbourhood $U_{q}$ of $q$ contained in $U_{p}$.

$N_{2}$. For each point $p$ and each pair of neighbourhoods $U_{p}$ and $W_{p}$ of $p$, there is a neighbourhood of $p$ contained in the intersection of $U_{p}$ and $W_{p}$.

The following are various $g$-topologies in a statistical metric space $(S, F)$ defined by Thorp [9].

(a) If the conditions $N_{0}$ and $N_{2}$ are satisfied, then the collection of neighbourhoods on $S$ is called the g-topology of type $V_{D}$.

(b) The collection of neighbourhoods on $S$ is called the g-topology of typeV $V_{\alpha}$ if the conditions $N_{0}$ and $N_{1}$ are satisfied.

(c) A $g$-topology is a topology if the conditions $N_{0}, N_{1}$ and $N_{2}$ are satisfied.

Let $S$ be a set and $P$ be a partially ordered $(<)$ set with least element 0 . A generalized écart [9] ( $g$-écart for short) is a mapping $G$ from $S \times S$ into $P$. If a $g$-écart $G$ satisfies $G(p, p)=0$ and the set $S$ consists of more than one point, the $g$-écart $g$-topology for $S$ is the $g$-topology determined from $G$, and its partially ordered range set $P$, as follows. For each $f>0$ in $P$ and each $p \in S$, the $f$-sphere for $p$ is $N_{p}(f)=\{q \in S \mid G(p, q)<f\}$. Then for each $p \in S$, the collection of $f$-spheres, $\mathscr{N}_{p}(P)=\left\{N_{p}(f) \mid f>0\right.$ in $\left.P\right\}$ is a family of neighbourhoods for $p$.

The $g$-écart associated with a statistical metric space $(S, F)$ is the mapping $G$ defined by $G(p, q)=G_{p q}$ [9].

Example 2.3. Let $S=\mathbb{N}$ and $P=\mathbb{N} \cup\{0\}$ be a partially ordered set with the relation $<$ where $\mathbb{N}$ denote the set of all natural numbers. Let $A=\{1,2,3\}$ be a subset of $S$. Define

$$
G(p, q)=\left\{\begin{array}{lll}
1 & \text { if } \quad p \notin A, q \in S, \\
1 & \text { if } \quad p \in S, q \notin A, . \\
\{0\} & \text { if } \quad p \notin A, q \notin A,
\end{array}\right.
$$

and $p \in A, q \in A$ define $G(p, q)$ as follows: $G(1,1)=0, G(1,2)=2, G(1,3)=3, G(2,1)=4, G(2,2)=0, G(2,3)=6, G(3,1)=1, G(3,2)=$ $2, G(3,3)=0$. Case 1: $p \notin A, q \in S$. Then $G(p, q)=1$. Let $f=1$. Then $N_{p}(1)=\emptyset$. For $f \geq 2, N_{p}(f)=S$. Case $2: p \in S, q \notin A$. Then 
$G(p, q)=1$. Let $f=1$. Then $N_{p}(1)=\emptyset$ and $N_{p}(f)=S-A$ for $f \geq 2$. Case $3: p \notin A, q \notin A$. Then $G(p, q)=0$ and so $N_{p}(f)=S-A$ for $f>0$. Case 4: $p \in A, q \in A$. Then $N_{1}(1)=N_{1}(2)=\{1\} ; N_{1}(3)=\{1,2\} ; N_{1}(f)=A$ for $f \geq 4$. Now $N_{2}(1)=N_{2}(2)=N_{2}(3)=N_{2}(4)=$ $\{2\} ; N_{2}(5)=N_{2}(6)=\{1,2\} ; N_{2}(f)=A$ for $f \geq 7$ and $N_{3}(1)=\{3\} ; N_{3}(2)=\{1,3\} ; N_{3}(f)=A$ for $f \geq 3$.

Given a statistical metric space $(S, F)$, for each pair of points $p$ and $r$ in $S$, the $r$-sphere with center $p, N_{p}(r)$ is defined to be the sphere $N_{p}\left(G_{p r}\right)=\left\{q \mid G_{p q}<G_{p r}\right\}$. The $R$-g-topology [9] for $(S, F)$ is the structure whose family of neighbourhoods at each point $p$ is the collection $\mathscr{N}_{p}(r)=\left\{N_{p}(r) \mid r \in S\right\}$.

Example 2.4. Consider the $\mathrm{SM}$ space $(S, F)$ where $S=\mathbb{N}$ and the distribution function

$$
F_{p q}(x)=\left\{\begin{array}{lll}
\frac{x}{d(p, q)} & \text { if } & 0<x<d(p, q), d(p, q) \neq 0 \\
1 & \text { if } & x \geq d(p, q)
\end{array} .\right.
$$

Fix $p=1$ and $r=2$ are in $S$. Let $x=\frac{1}{4}$. Then $G_{p r}(x)=0.75$. Now $N_{1}(0.75)=\{1\}$.

Observation 2.5. In a statistical metric space, $N_{p}\left(G_{p r}\right)=\emptyset$ if $p=r$.

Notations 2.6. In a $S M$ space $(S, F)$, we use the following notations:

(a) Let $\tau$ denote the $g$-topology of typeV.

(b) Let $\tau_{D}$ denote the $g$-topology of type $V_{D}$.

(c) Let $\tau_{\alpha}$ denote the $g$-topology of type $V_{\alpha}$.

(d) Let $\tau_{e}$ denote the $g$-écart $g$-topology.

(e) Let $\tau_{R}$ denote the $R$-g-topology.

(f) Each element in $\mathscr{N}(X)$ is called a $\tau$-neighborhood.

(f) Each element in $\mathscr{N}_{p}(P)$ is called a $\tau_{e}$-neighborhood.

(f) Each element in $\mathscr{N}_{p}(r)$ is called a $\tau_{R}$-neighborhood.

\section{Behaviour of various $g$-topology}

In this section, we give some properties and find the relations between four types of neighborhoods in a SM space. Also, we give the answer for some of the questions raised by Throp [9].

Theorem 3.1. Let $(S, F)$ be a statistical metric space. Then the following hold.

(a) If $u_{1} \leq u$ and $v_{1} \leq v$, then $N_{p}\left(u_{1}, v_{1}\right) \subset N_{p}(u, v)$ where $u, v, u_{1}, v_{1}>0$.

(b) If $\kappa=\left\{\mathscr{N}(Z), \mathscr{U}(Z), \mathscr{N}_{p}(P), \mathscr{N}_{p}(r)\right\}$ and $A \in \kappa$, then there exist $B, C \in \kappa$ such that $B \subset A \subset C$.

Proof.

(a) Let $q \in N_{p}\left(u_{1}, v_{1}\right)$. Then $F_{p q}\left(u_{1}\right)>1-v_{1}$. Since $u_{1} \leq u$ and $v_{1} \leq v, F_{p q}(u) \geq F_{p q}\left(u_{1}\right)>1-v_{1} \geq 1-v$. Thus, $F_{p q}(u)>1-v$. Therefore, $q \in N_{p}(u, v)$. Hence $N_{p}\left(u_{1}, v_{1}\right) \subset N_{p}(u, v)$.

(b) We give the detailed proof only for $\kappa=\mathscr{N}(Z)$ and $\kappa=\mathscr{N}_{p}(r)$. Suppose that $\kappa=\mathscr{N}(Z)$ and $A \in \kappa$. Then $A=N_{p}(u, v)$ where $u, v>0$. Take $0<u_{1} \leq u, 0<v_{1} \leq v$ and $B=N_{p}\left(u_{1}, v_{1}\right)$. By (a), $B \subset A$. If $u_{2} \geq u, v_{2} \geq v$, then $u_{2}>0, v_{2}>0$. Define $C=N_{p}\left(u_{2}, v_{2}\right)$. By (a), $A \subset C$. Thus, there exist $B, C \in \mathscr{N}(Z)$ such that $B \subset A \subset C$.

Suppose that $\kappa=\mathscr{N}_{p}(r)$. Let $A \in \kappa$. Then $A=\left\{q \in S \mid G_{p q}<G_{p r}\right\}$ and so $A=\left\{q \in S \mid G_{p q}(u)<G_{p r}(u)\right\}$ where $u>0$. Take $u_{1} \geq u$. Define $B=\left\{q \in S \mid G_{p q}(u)<G_{p r}\left(u_{1}\right)\right\}$. Then $B \in \mathscr{N}_{p}(r)$. Let $s \in B$. Then $G_{p s}(u)<G_{p r}\left(u_{1}\right)$ and so $G_{p s}(u)<G_{p r}(u)$, since $G_{p r}\left(u_{1}\right) \leq G_{p r}(u)$. Therefore, $s \in A$. Hence $B \subset A$. Define $C=\left\{q \in S \mid G_{p q}\left(u_{1}\right)<G_{p r}(u)\right\}$. Then $C \in \mathscr{N}_{p}(r)$. Let $s \in A$. Then $G_{p s}(u)<G_{p r}(u)$ and so $G_{p s}\left(u_{1}\right)<G_{p r}(u)$, since $G_{p s}\left(u_{1}\right) \leq G_{p s}(u)$. Therefore, $s \in C$. Hence $A \subset C$.

From the definition of $g$-topology of $t y p e V_{D}$, it is observed that every $g$-topological space of type $V_{D}$ is a $g$-topological space of $t y p e V$. The following Theorem 3.2 discusses the converse of the question that "What are the necessary and sufficient conditions that the $g$-topology of typeV to be of typeV $V_{D}$ ?" which is raised by Thorp [9].

Theorem 3.2. Let $(S, F)$ be a statistical metric space. Then the following hold.

(a) $\tau$ satisfies $\mathrm{N}_{2}$.

(b) The g-topology of typeV is a g-topology of type $V_{D}$.

Proof. (a) Let $U_{p}$ and $W_{p}$ be two neighbourhoods of $p$. Then $U_{p}=\left\{q \in S \mid G_{p q}(u)<v\right\}$ and $W_{p}=\left\{q \in S \mid G_{p q}\left(u_{1}\right)<v_{1}\right\}$. Define $V_{p}=\left\{q \in S \mid G_{p q}\left(\min \left(u, u_{1}\right)\right)<\min \left(v, v_{1}\right)\right\}$. Then $p \in V_{p}$ and so $V_{p}$ is a neighbourhood of $p$. Since $\min \left(u, u_{1}\right) \leq u, \min \left(v, v_{1}\right) \leq v$, 
we have $V_{p} \subset U_{p}$, by Theorem 3.1(a). Also, $\min \left(u, u_{1}\right) \leq u_{1}$ and $\min \left(v, v_{1}\right) \leq v_{1}$. Therefore, $V_{p} \subset W_{p}$, by Theorem 3.1(a). Hence $V_{p} \subset U_{p} \cap W_{p}$. Therefore, $\tau$ satisfies $N_{2}$.

(b) By (a) and the definition of $g$-topology of $\operatorname{type}_{D}$, it follows that every $g$-topology of typeV is a $g$-topology of type $V_{D}$.

The following two questions are raised by Thorp [9].

(I) "What are the necessary and sufficient conditions that the $g$-topology of $\operatorname{typeV}_{\alpha}$ to be the $g$-topology of typeV $V_{D}$ ?".

(II) "What conditions are both necessary and sufficient for the $g$-topology of $t y p e V_{\alpha}$ to be a topology?".

The following Corollary 3.3 (a) gives a necessary condition for the given space to be a $g$-topological space of $t y p e V_{D}$ which also gives a partial answer to the question (I) and Corollary 3.3 (b) gives the answer to the question (II).

Corollary 3.3. Let $(S, F)$ be a statistical metric space. Then the following hold.

(a) The g-topology of type $V_{\alpha}$ is a g-topology of type $V_{D}$.

(b) The g-topology of type $V_{\alpha}$ is a topology and conversely.

Proof. (a) By the definition of $g$-topology of $t y p e V_{\alpha}, g$-topology of $t y p e V_{\alpha}$ is a $g$-topology of typeV. Therefore, $g$-topology of typeV $V_{\alpha}$ is a $g$-topology of typeV $_{D}$, by Theorem 3.2(b).

(b) By the definition of $g$-topology of $t y p e V_{\alpha}, g$-topology of type $V_{\alpha}$ is a $g$-topology of typeV and satisfies the condition $N_{1}$. By (a), $g$-topology of type $V_{\alpha}$ satisfies the condition $N_{2}$, by the definition $g$-topology of $t y p e V_{D}$. Hence a $g$-topology of type $V_{\alpha}$ is a topology. Converse follows from the definition of topology in a statistical metric space.

Theorem 3.4. Let $(S, F)$ be a statistical metric space. If $U \in \mathscr{U}(Z)$, then there exists $V \subset S$ such that $V \in \mathscr{N}(Z)$.

Proof. Let $U \in \mathscr{U}(Z)$. Define $V=\{q \in S \mid(p, q) \in U\}$. Since $U \in \mathscr{U}(Z), V=\left\{q \in S \mid G_{p q}(u)<v\right\}$. Hence $V=N_{p}(u, v)$, by the definition of $N_{p}(u, v)$. Therefore, $V \in \mathscr{N}(Z)$.

Theorem 3.5. Let $(S, F)$ be a statistical metric space. Then the following hold.

(a) $\tau_{e} \subset \tau$

(b) $\tau_{e}$ satisfies $N_{2}$.

Proof. (a) Let $p \in S$ and $U$ be a $\tau_{e}$-neighbourhood of a point $p$. Then $U=\{q \in S \mid G(p, q)<f\}$. Since in a statistical metric space $G(p, q)=G_{p q}, U=\left\{q \in S \mid G_{p q}<f\right\}$. Here $0<f \in P$ where $P$ is a partially ordered set. Then there is an element $g \in P$ such that $g<f$. Take $g(u)=v$ for all $u>0$. Then $v>0$. Define $V=\left\{q \in S \mid G_{p q}(u)<v\right\}$. Then $V$ is a $\tau$-neighbourhood such that $p \in V \subset U$. Hence $\tau_{e} \subset \tau$.

(b) Suppose that $U_{p}$ and $W_{p}$ are two neighbourhoods of $p$. Then $U_{p}=\left\{q \in S \mid G(p, q)<f_{1}\right\}$ and $W_{p}=\left\{q \in S \mid G(p, q)<f_{2}\right\}$. Consider $V_{p}=\left\{q \in S \mid G(p, q)<\min \left(f_{1}, f_{2}\right)\right\}$. Then $p \in V_{p}$ and so $V_{p}$ is a neighbourhood of $p$. Also, $V_{p} \subset U_{p} \cap W_{p}$. Therefore, $\tau_{e}$ satisfies $N_{2}$.

The following Corollary 3.6 gives a necessary condition for the given space to be a $g$-topological space of $\operatorname{typeV}_{D}$ which also gives a partial answer to the question that "What are the necessary and sufficient conditions for $\tau_{e}$ to be of $t y p e V_{D}$ ?" raised by Thorp [9].

Corollary 3.6. Let $(S, F)$ be a statistical metric space. Then $\tau_{e} \subset \tau_{D}$.

Proof. Follows from Theorem 3.5 and the definition of $\operatorname{typeV}_{D}$

Theorem 3.7. Let $(S, F)$ be a statistical metric space. Then the following hold.

(a) $\tau_{R}$ satisfies $N_{2}$.

(b) $\tau_{R} \subset \tau$.

Proof. (a) Let $U_{p}$ and $W_{p}$ be $\tau_{R}$-neighbourhoods of $p$. Then $U_{p}=\left\{q \in S \mid G_{p q}<G_{p r_{1}}\right\}$ and $W_{p}=\left\{q \in S \mid G_{p q}<G_{p r_{2}}\right\}$ where $r_{1}, r_{2} \in S$. Define $V_{p}=\left\{q \in S \mid G_{p q}<\inf \left(G_{p r_{1}}, G_{p r_{2}}\right)\right\}$. It follows that $V_{p}$ is a neighbourhood of $p$ and $p \in V_{p}$. Also, $V_{p} \subset U_{p} \cap W_{p}$. Therefore, $\tau_{R}$ satisfies $N_{2}$. 
(b) If $t \in S$ and $B$ is a $\tau_{R}$-neighbourhood of $t$, then $B=\left\{q \mid G_{p q}<G_{p r}\right\}$ and so $B=\left\{q \mid G_{p q}(u)<G_{p r}(u)\right\}$ where $u>0$. Choose an element $v$ such that $u<v$. Take $v_{1}=G_{p r}(v)$. Since $B \neq \emptyset$ we have $p \neq r$ and so $G_{p r}(v) \neq 0$ so that $v_{1}>0$. Define $B_{1}=\left\{q \mid G_{p q}(u)<v_{1}\right\}$.

Then $t \in B_{1}$ and $B_{1}$ is a $\tau$-neighbourhood contained in $B$. Hence $\tau_{R} \subset \tau$.

The following Corollary 3.8 gives a necessary condition for the given space to be a $g$-topological space of $t y p e V_{D}$ which also gives a partial answer to the question that "What are the necessary and sufficient conditions for the $R$ - $g$-topology to be $g$-topology of $t y p e V_{D}$ ?" raised by Thorp in [9].

Corollary 3.8. Let $(S, F)$ be a statistical metric space. Then $\tau_{R} \subset \tau_{D}$.

Proof. Follows from Theorem 3.7 and the definition of type $V_{D}$.

Lemma 3.9. A function $T: I \times I \rightarrow I$ is defined by $T(x, y)=\max (x, y)$ where $I=[0,1]$. Then $T$ satisfies the conditions $(T-I I)$ and $(T$-IV).

Proof. (a) Suppose that $c \geq a, d \geq b$ where $a, b, c, d \in[0,1]$. Now $T(c, d)=\max (c, d)$ and $T(a, b)=\max (a, b)$. Case-1: If $T(c, d)=c$ and $T(a, b)=a$, then $T(c, d) \geq T(a, b)$. Case-2: Suppose $T(c, d)=c$ and $T(a, b)=b$. Since $b \leq d \leq c, T(c, d) \geq T(a, b)$. Case-3: If $T(c, d)=d$ and $T(a, b)=b$, then $T(c, d) \geq T(a, b)$. Case-4: Suppose $T(c, d)=d$ and $T(a, b)=a$. Since $a \leq c \leq d, T(c, d) \geq T(a, b)$. Therefore, $T$ satisfies the condition (T-II).

(b) Now $T(1,1)=\max (1,1)=1$. Hence $T$ satisfies the condition (T-IV).

The following Theorem 3.10 gives the answer to the question that "What are the necessary and sufficient conditions for the $g$-topology of typeV to be a topology?" raised by Thorp in [9].

Theorem 3.10. Let $(S, F)$ be a statistical metric space with the g-topology of typeV. If SM-IVm satisfies under $T: I \times I \rightarrow I$ defined by $T(x, y)=\max (x, y)$, then the $g$-topology on $S$ is a topology and conversely.

Proof. Given that $(S, F)$ is a statistical metric space with a $g$-topology of typeV. Then by Theorem 3.2, $N_{0}$ and $N_{2}$ are satisfied. Let $p \in S$ and $U_{p}$ be a neighbourhood for $p$. Then $U_{p}=\left\{r \in S \mid F_{p r}(u)>1-v\right\}$. Choose $u_{1}=\frac{u}{2}$ and $v_{1}<v$ with $0 \leq v_{1} \leq 1$. Taking $W_{p}=\{s \in S \mid$ $\left.F_{p s}\left(u_{1}\right)>1-v_{1}\right\}$, we get that $W_{p}$ is a neighbourhood of $p$. For $q \in W_{p}$, define $V_{q}=\left\{t \in S \mid F_{q t}\left(u_{1}\right)>1-v_{1}\right\}$ so that $V_{q}$ is a neighbourhood of $q$. Since $q \in W_{p}, F_{p q}\left(u_{1}\right)>1-v_{1}$ and so $F_{q p}\left(u_{1}\right)>1-v_{1}$, by the condition (SM-III). Hence $p \in V_{q}$. If $a \in V_{q}$, then $F_{q a}\left(u_{1}\right)>1-v_{1}$. Since $p \in V_{q}, F_{q p}\left(u_{1}\right)>1-v_{1}$. By Lemma 3.9, $T$ satisfies the condition (T-II). Thus, $T\left(F_{p q}\left(u_{1}\right), F_{q a}\left(u_{1}\right)\right) \geq T\left(1-v_{1}, 1-v_{1}\right)$. By $(\mathrm{SM}-\mathrm{IVm})$, $F_{p a}(u) \geq T\left(F_{p q}\left(u_{1}\right), F_{q a}\left(u_{1}\right)\right)$, since $u_{1}=\frac{u}{2}$ which implies that $F_{p a}(u) \geq T\left(1-v_{1}, 1-v_{1}\right)$ which in turn implies that $F_{p a}(u) \geq 1-v_{1}$, by hypothesis. Hence $F_{p a}(u)>1-v$ and so $a \in U_{p}$. Therefore, $V_{q} \subset U_{p}$ and consequently $N_{1}$ is satisfied. Thus, $g$-topology of $t y p e V$ is a topology. Converse part follows from the definition of topology in a statistical metric space.

The following Corollary 3.11 gives the answer to the question "What are the necessary and sufficient conditions for the $g$-topology of typeV $_{D}$ to be a topology?" raised by Thorp [9].

Corollary 3.11. Let $(S, F)$ be a statistical metric space with the g-topology of typeV $V_{D}$. If SM-IVm satisfies under a function $T: I \times I \rightarrow I$ defined by $T(x, y)=\max (x, y)$, then the g-topology of type $V_{D}$ is a topology and conversely.

Proof. By the definition of $t y p e V_{D}$, it follows that it is of typeV. By hypothesis and Theorem 3.10, g-topology of type $V_{D}$ is a topology. Converse follows from the definition of topology in a statistical metric space.

The following Corollary 3.12 gives a sufficient condition for $g$-topology of $t y p e V_{D}$ to be a $g$-topology of $t y p e V_{\alpha}$ which also gives a partial answer to the question "What conditions are both necessary and sufficient for the g-topology of typeV $V_{\alpha}$ to be of typeV $V_{D}$ ?" raised by Thorp in [9].

Corollary 3.12. Let $(S, F)$ be a statistical metric space with the g-topology of typeV $V_{D}$. If SM-IVm satisfies under a function $T: I \times I \rightarrow I$ defined by $T(x, y)=\max (x, y)$, then $g$-topology of type $V_{D}$ is a g-topology of type $V_{\alpha}$.

Proof. By the definition of $t y p e V_{D}, g$-topology of $t y p e V_{D}$ is of typeV. As in the proof of Theorem 3.10, typeV satisfies the condition $N_{1}$. Therefore, $g$-topology of $t y p e V_{D}$ is a $g$-topology of $t y p e V_{\alpha}$.

The following Theorem 3.13 gives a necessary condition for the $g$-écart- $g$-topology to be a topology which also gives a partial answer to the questions "What are the necessary and sufficient conditions for the $g$-écart- $g$-topology to be a topology?" raised by Thorp [9].

Theorem 3.13. Let $(S, F)$ be a statistical metric space with g-écart-g-topology. If SM-IVm holds under a function T satisfying T-IV, T-II and sup $_{x<1} T(x, x)=1$, then the g-écart $g$-topology is a topology on $S$.

Proof. By Corollary 3.6, g-écart $g$-topology is a $g$-topology of type $V_{D}$ and hence the conditions $N_{0}$ and $N_{2}$ are satisfied. Let $p \in S$ and $U_{p}$ be a neighbourhood of $p$. Then $U_{p}=\left\{r \in S \mid G_{p r}<f\right\}$. Let $f_{1}$ be a tail with $L<f_{1}<f$. If $W_{p}=\left\{s \in S \mid G_{p s}<f_{1}\right\}$, then $W_{p}$ is a neighbourhood of $p$. Choose $q \in W_{p}$ and take $V_{q}=\left\{t \in S \mid G_{q t}<f_{1}\right\}$. Then $V_{q}$ is a neighbourhood of $q$. Since $q \in W_{p}, G_{p q}<f_{1}$ and so $G_{q p}<f_{1}$ which implies that $p \in V_{q}$ which in turn implies that $G_{q p}<f_{1}$ and hence $F_{q p}(x)>1-f_{1}(x)$. Let $m \in V_{q}$. Then $G_{q m}<f_{1}$ and so $F_{q m}(x)>1-f_{1}(x)$. By T-II, $T\left(F_{p q}(x), F_{q m}(x)\right) \geq T\left(1-f_{1}(x), 1-f_{1}(x)\right)$. Also, $F_{p m}(2 x) \geq T\left(F_{p q}(x), F_{q m}(x)\right)$, by SM-IVm. Hence it 
suffices to find a $f_{1}$ such that $T\left(1-f_{1}(x), 1-f_{1}(x)\right) \geq 1-f_{1}(2 x)$ for some $x$. Since $f>L$, there exists $a>0$ such that $1-f(2 a)<1$. By hypothesis, there is a number $b<1$ such that $T(b, b)>1-f(2 a)$. Now we define $f_{1}(x)$ using $a$ and $b$ by

$$
f_{1}(x)=\left\{\begin{array}{rll}
0 & \text { if } & x>a \\
1-b & \text { if } & 0<x \leq a .
\end{array}\right.
$$

If $x>a$, then $T\left(1-f_{1}(x), 1-f_{1}(x)\right)=T(1,1)$. Again, using T-IV, $T\left(1-f_{1}(x), 1-f_{1}(x)\right)=1$. Therefore, $T\left(1-f_{1}(x), 1-f_{1}(x)\right) \geq$ $1-f(2 x)$. If $0<x \leq a$, then $T\left(1-f_{1}(x), 1-f_{1}(x)\right)=T(b, b)>1-f(2 a) \geq 1-f(2 x)$, since $f$ is a left continuous function. Thus, $T\left(1-f_{1}(x), 1-f_{1}(x)\right)>1-f(2 x)$ for $0<x \leq a$. Hence $F_{p m}(2 x)>1-f(2 x)$ for $0<x \leq a$. Thus, $m \in U_{p}$ so that $V_{q} \subset U_{p}$. Therefore, $N_{1}$ is satisfied and hence $g$-écart $g$-topology is a topology.

Theorem 3.14 below gives a necessary condition for an $R$ - $g$-topology to be a topology which also gives a partial answer to the question "What are the necessary and sufficient conditions for the $R$ - $g$-topology to be a topology?" raised by Thorp in [9].

Theorem 3.14. Let $(S, F)$ be a statistical metric space with $R$-g-topology. If SM-IVm satisfies under a function $T: I \times I \rightarrow I$ defined by $T(x, y)=\max (x, y)$, then the R-g-topology is a topology.

Proof. By hypothesis and Corollary 3.8, $R$ - $g$-topology is a $g$-topology of $t y p e V_{D}$ and hence the conditions $N_{0}$ and $N_{2}$ are satisfied. Let $p \in S$ and $U_{p}$ be a neighbourhood for $p$. Then $U_{p}=\left\{s \in S \mid G_{p s}<G_{p r}\right\}$. Take $0<c \leq 1$ and define $W_{p}=\left\{t \in S \mid G_{p t}<c G_{p r}\right\}$. Then $W_{p}$ is a neighbourhood of $p$. If $q \in W_{p}$, then $G_{p q}<c G_{p r}$ and so $G_{q p}<c G_{p r}$. Hence $p \in\left\{u \in S \mid G_{q u}<c G_{p r}\right\}$. Take $V_{q}=\left\{u \in S \mid G_{q u}<c G_{p r}\right\}$. Then $p \in V_{q}$ and $V_{q}$ is a neighbourhood of $q$. If $n \in V_{q}$, then $G_{q n}<c G_{p r}$ and so $G_{q n}<G_{p r}$ so That $F_{q n}>F_{p r}$. Since $p \in V_{q}, G_{q p}<c G_{p r}<G_{p r}$ and hence $F_{q p}>F_{p r}$. By SM-IVm, $F_{p n}(x) \geq T\left(F_{p q}(0), F_{q n}(x)\right)=\operatorname{Max}\left(0, F_{q n}(x)\right)=F_{q n}(x)$, by hypothesis and SM-II. Thus, $F_{p n}(x) \geq F_{q n}(x)$ so that $F_{p n}(x)>F_{p r}(x)$ and hence $G_{p n}<G_{p r}$. Therefore, $n \in U_{p}$ and so $V_{q} \subset U_{p}$. Thus, $N_{1}$ is satisfied. Therefore, the $R$ - $g$-topology is a topology.

In [6], Min introduced stack as in the following way: A collection $\mathscr{C}$ of subsets of $S$ is called a stack [6] if $A \in \mathscr{C}$ whenever $B \in \mathscr{C}$ and $B \subset A$. Also, he analyzes whether a neighbourhood collections are stack or not in generalized topological spaces. Here we prove that different types of the neighbourhood collections become stack in statistical metric spaces.

Theorem 3.15. Let $(S, F)$ be a statistical metric space. Then $\mathscr{N}(Z)$ is a stack.

Proof. Let $A \in \mathscr{N}(Z)$ and $A \subseteq B$. Then $A=\left\{q \in S \mid G_{p q}(u)<v\right\}$. Take $u_{1}>u$ and

$$
v_{1}=\left\{\begin{array}{c}
v \text { if } \quad s \in A, \\
G_{p s}(u) \text { if } \quad s \in B-A, \\
G_{p s}\left(u_{1}\right) \text { if } \quad s \in S-B .
\end{array}\right.
$$

Then $u_{1}>0$ and $v_{1}>0$. If $U=\left\{q \in S \mid G_{p q}\left(u_{1}\right)<v_{1}\right\}$, then $U \in \mathscr{N}(Z)$. Choose $t \in B$. Then $t \in A$ or $t \in B-A$. Suppose $t \in A$. Then $G_{p t}(u)<v$. Since $u_{1}>u, G_{p t}\left(u_{1}\right)<G_{p t}(u)$ which implies that $G_{p t}\left(u_{1}\right)<v=v_{1}$ and hence $t \in U$. If $t \in B-A$, then $G_{p t}(u)>v$. Since $u_{1}>u, G_{p t}\left(u_{1}\right)<G_{p t}(u)=v_{1}$ and so $t \in U$. Hence $B \subset U$. Let $s \in U$. Then $G_{p s}\left(u_{1}\right)<v_{1}$. By the definition of $v_{1}, s \in A$ or $s \in B-A$. This implies that $s \in B$ which implies that $U \subset B$. Therefore, $B=U$. Since $U \in \mathscr{N}(Z), B \in \mathscr{N}(Z)$. Hence $\mathscr{N}(Z)$ is a stack.

Theorem 3.16. Let $(S, F)$ be a statistical metric space. Then $\mathscr{U}(Z)$ is a stack.

Proof. Let $A \in \mathscr{U}(Z)$ and $A \subseteq B$. Then $A=\left\{(p, q) \in S \times S \mid G_{p q}(u)<v\right\}$. Take $u_{1}>u$ and

$$
v_{1}=\left\{\begin{array}{c}
v \text { if } \quad(p, q) \in A, \\
G_{p q}(u) \text { if } \quad(p, q) \in B-A, \\
G_{p q}\left(u_{1}\right) \quad \text { if } \quad(p, q) \in S-B .
\end{array}\right.
$$

Then $u_{1}$ and $v_{1}>0$. Define $U=\left\{(p, q) \in S \times S \mid G_{p q}\left(u_{1}\right)<v_{1}\right\}$ so that $U \in \mathscr{U}(Z)$. If $(s, t) \in B$, then $(s, t) \in A$ or $(s, t) \in B-A$. If $(s, t) \in A$, then $G_{s t}(u)<v$. Since $u_{1}>u, G_{s t}\left(u_{1}\right)<G_{s t}(u)$ which implies that $G_{s t}\left(u_{1}\right)<v=v_{1}$ and hence $(s, t) \in U$. Suppose that $(s, t) \in B-A$. Then $G_{s t}(u)>v$. Since $u_{1}>u, G_{s t}\left(u_{1}\right)<G_{s t}(u)=v_{1}$ and so $(s, t) \in U$. Hence $B \subset U$. Let $(l, m) \in U$. Then $G_{l m}\left(u_{1}\right)<v_{1}$. By definition of $v_{1},(l, m) \in A$ or $(l, m) \in B-A$. This implies that $(l, m) \in B$ which implies that $U \subset B$. Therefore, $B=U$. Since $U \in \mathscr{U}(Z), B \in \mathscr{U}(Z)$. Hence $\mathscr{U}(Z)$ is a stack.

Theorem 3.17. Let $(S, F)$ be a statistical metric space. Then $\mathscr{N}_{p}(P)$ is a stack.

Proof. Let $A \in \mathscr{N}_{p}(P)$ and $A \subseteq B$. Then $A=\{q \in S \mid G(p, q)<f\}$. In a statistical metric space, $G_{p q}=G(p, q)$ so that $A=\left\{q \in S \mid G_{p q}(u)<\right.$ $f(u)\}$ where $u>0$. Take $u_{1}>u$ and

$$
f_{1}\left(u_{1}\right)=\left\{\begin{array}{c}
f(u) \quad \text { if } \quad s \in A, \\
G_{p s}(u) \text { if } \quad s \in B-A, \\
G_{p s}\left(u_{1}\right) \text { if } \quad s \in S-B
\end{array}\right.
$$

Define $U=\left\{q \in S \mid G(p, q)<f_{1}\right\}$. Then $U \in \mathscr{N}_{p}(P)$. Since $(S, F)$ is a statistical metric space, $U=\left\{q \in S \mid G_{p q}\left(u_{1}\right)<f_{1}\left(u_{1}\right)\right\}$. Let $t \in B$. Then $t \in A$ or $t \in B-A$. If $t \in A$, then $G_{p t}(u)<f(u)$. Since $u_{1}>u, G_{p t}\left(u_{1}\right)<G_{p t}(u)$ which implies that $G_{p t}\left(u_{1}\right)<f(u)=f_{1}\left(u_{1}\right)$ and hence $t \in U$. If $t \in B-A$, then $G_{p t}(u)>v$. Since $u_{1}>u, G_{p t}\left(u_{1}\right)<G_{p t}(u)=f_{1}\left(u_{1}\right)$ and so $t \in U$. Hence $B \subset U$. Let $s \in U$. Then $G_{p s}\left(u_{1}\right)<f_{1}\left(u_{1}\right)$. By definition of $v_{1}, s \in A$ or $s \in B-A$. This implies that $s \in B$ which implies that $U \subset B$. Therefore, $B=U$ and so $B \in \mathscr{N}_{p}(P)$, since $U \in \mathscr{N}_{p}(P)$. Hence $\mathscr{N}_{p}(P)$ is a stack.

The following Theorem 3.18 shows that a neighbourhood collection $\mathscr{N}(Z)$ is closed under finite intersection in a statistical metric space. 
Theorem 3.18. Let $(S, F)$ be a statistical metric space and $\kappa=\left\{\mathscr{N}(Z), \mathscr{N}_{p}(P)\right\}$. If $W_{1}, W_{2}, \ldots, W_{n} \in Q$ with $W_{1} \cap W_{2} \cap \ldots \cap W_{n} \neq \emptyset$, then $W_{1} \cap W_{2} \cap \ldots \cap W_{n} \in Q$ where $Q \in \kappa$.

Proof. We will give a detailed proof only for $Q=\mathscr{N}(Z)$ where $Q \in \kappa$. Suppose that $V_{1}, V_{2}, \ldots, V_{n} \in Q$ with $V_{1} \cap V_{2} \cap \ldots \cap V_{n} \neq \emptyset$. Let $x \in V_{1} \cap V_{2} \cap \ldots \cap V_{n}$. Then $x \in V_{i}$ for $i=1$ to $n$. Since $V_{1}$ and $V_{2}$ are $\tau$-neighbourhoods containing $x$, there exists $\tau$-neighbourhood $W_{1}$ containing $x$ such that $W_{1} \subset V_{1} \cap V_{2}$, by Theorem 3.2(a). Again, $W_{1}$ and $V_{3}$ are $\tau$-neighbourhoods containing $x$ implies that there exists a $\tau$ neighbourhood $W_{2}$ containing $x$ such that $W_{2} \subset W_{1} \cap V_{3} \subset V_{1} \cap V_{2} \cap V_{3}$. Proceeding like this, we get a $\tau$-neighbourhood $W_{n-1}$ containing $x$ such that $W_{n-1} \subset W_{n-2} \cap V_{n} \subset V_{1} \cap V_{2} \cap V_{3} \cap \ldots \cap V_{n}$. Since $W_{n-1} \in Q$ and $W_{n-1} \subset V_{1} \cap V_{2} \cap V_{3} \cap \ldots \cap \cap V_{n}$ we have $V_{1} \cap V_{2} \cap V_{3} \cap \ldots \cap V_{n} \in Q$, by Theorem 3.15 .

\section{Relation between GMS and SM space}

In this section, we find the relations between $\lambda_{\Omega}$-open sets in generalized metric spaces and various $g$-topology neighbourhoods defined in statistical metric spaces. Also, we give some properties of $\lambda_{\Omega}$-open sets, kernel and perfect kernel in generalized metric spaces.

The notion of a generalized metric space was introduced by Korczak-Kubiak et al. in [2]. Let $X \neq \emptyset$. The symbol $\Omega$ to denote the family consisting of metrics defined on subsets of $X$, that is, if $\rho \in \Omega$, then there exists a non-null set $A_{\rho} \subset X$ such that $\rho$ is a metric on $A_{\rho}$ where $A_{\rho}$ is a domain of $\rho$ and it will be denoted by $\operatorname{dom}(\rho)$. The space $(X, \Omega)$ is called a generalized metric space (GMS) [2]. We will write $\Omega_{X}$ if we want to point out that all the metrics $\rho \in \Omega_{X}$ defined on $X$ [2].

Denote $\lambda_{\Omega}$ is the family of $\Omega$-open sets in $(X, \Omega)$, more precisely, $V \in \lambda_{\Omega}$ if and only if for each $x \in V$, there exist $\rho \in \Omega$ and $\varepsilon>0$ such that $B_{\rho}(x, \varepsilon) \subset V$ where $B_{\rho}(x, \varepsilon)=\{y \in \operatorname{dom}(\rho): \rho(x, y)<\varepsilon\}[2]$.

Let $(X, \Omega)$ be a GMS. A kernel [2] of the space $(X, \Omega)$ is a finite family $\Omega_{0} \subset \Omega$ with the following property: for any set $V \in \tilde{\lambda}_{\Omega}$, there exists $\rho \in \Omega_{0}$ such that $i_{\rho} V \neq \emptyset$. A finite family $\Omega_{0} \subset \Omega$ is called a perfect kernel [2] of the space $(X, \Omega)$ if for any $V_{1}, V_{2}, \ldots, V_{m} \in \mu_{\Omega}$ such that $V_{1} \cap V_{2} \cap \ldots \cap V_{m} \neq \emptyset$, there exists $\rho \in \Omega_{0}$ such that $i_{\rho}\left(V_{1} \cap V_{2} \cap \ldots \cap V_{m}\right) \neq \emptyset$ [2]. Every perfect kernel is a kernel [2].

A GMS $(X, \Omega)$ is said to be a weakly complete space [2] if there exists a kernel $\Omega_{0} \subset \Omega$ consisting of complete metrics. A GMS (X, $\Omega$ ) is said to be a complete space [2] if there exists a perfect kernel $\Omega_{0} \subset \Omega$ consisting of complete metrics. Every complete space is a weakly complete space [2].

Definition 4.1. Let $(X, \Omega)$ be a generalized metric space. Then $\Omega$ is said to satisfy $\mathscr{V}$-property if $\sigma_{1}, \sigma_{2} \in \Omega$ and $x, y \in X$, then $\sigma(x, y)=$ $\max \left\{\sigma_{1}(x, y), \sigma_{2}(x, y)\right\}$ is a metric and hence $\sigma \in \Omega$.

Theorem 4.2. Let $(X, \Omega)$ be a generalized metric space. Then $\lambda_{\Omega}$ satisfies the condition $N_{1}$.

Proof. Let $p \in X$ and $U_{p}$ be a neighbourhood of $p$. Then $U_{p} \in \tilde{\lambda}_{\Omega}$. Since $p \in U_{p}$, there is a metric $\sigma_{1} \in \Omega$ and $\varepsilon_{1}>0$ such that $B_{\sigma_{1}}\left(p, \varepsilon_{1}\right) \subset U_{p}$. Since $B_{\sigma_{1}}\left(p, \varepsilon_{1}\right) \in \tilde{\lambda}_{\Omega}$, for every $q \in B_{\sigma_{1}}\left(p, \varepsilon_{1}\right)$, there exist $\sigma \in \Omega$ and $\varepsilon>0$ such that $B_{\sigma}(q, \varepsilon) \subset B_{\sigma_{1}}\left(p, \varepsilon_{1}\right) \subset U_{p}$. Therefore, every $\lambda_{\Omega}$ satisfies the condition $N_{1}$.

Theorem 4.3. Let $(X, \Omega)$ be a generalized metric space and $\Omega$ satisfies the $\mathscr{V}$-property. Then the following hold.

(a) $\lambda_{\Omega}$ satisfies $N_{2}$.

(b) If $W_{1}, W_{2}, \ldots, W_{n} \in \lambda_{\Omega}$ with $W_{1} \cap W_{2} \cap \ldots \cap W_{n} \neq \emptyset$, then $W_{1} \cap W_{2} \cap \ldots \cap W_{n} \in \lambda_{\Omega}$.

Proof. (a) Let $p \in X$ and $U_{p}, W_{p} \in \lambda_{\Omega}$. Then there exist $\sigma_{1}, \sigma_{2} \in \Omega$ and $\varepsilon_{1}, \varepsilon_{2}>0$ such that $B_{\sigma_{1}}\left(p, \varepsilon_{1}\right) \subset U_{p}, B_{\sigma_{2}}\left(p, \varepsilon_{2}\right) \subset W_{p}$. For $y \in X$, define $\sigma_{3}(x, y)=\max \left\{\sigma_{1}(x, y), \sigma_{2}(x, y)\right\}$. Then $\sigma_{3} \in \Omega$ and $\sigma_{3}(x, y) \geq \sigma_{1}(x, y), \sigma_{3}(x, y) \geq \sigma_{2}(x, y)$. This implies that $B_{\sigma_{3}}\left(p, \varepsilon_{1}\right) \subset$ $B_{\sigma_{1}}\left(p, \varepsilon_{1}\right)$ and $B_{\sigma_{3}}\left(p, \varepsilon_{2}\right) \subset B_{\sigma_{2}}\left(p, \varepsilon_{2}\right)$ which implies that $B_{\sigma_{3}}\left(p, \varepsilon_{1}\right) \cap B_{\sigma_{3}}\left(p, \varepsilon_{2}\right) \subset B_{\sigma_{1}}\left(p, \varepsilon_{1}\right) \cap B_{\sigma_{2}}\left(p, \varepsilon_{2}\right)$. Choose $\varepsilon=\min \left\{\varepsilon_{1}, \varepsilon_{2}\right\}$ so that $\varepsilon>0$. Then $B_{\sigma_{3}}(p, \varepsilon) \subset B_{\sigma_{3}}\left(p, \varepsilon_{1}\right) \cap B_{\sigma_{3}}\left(p, \varepsilon_{2}\right)$ and so $B_{\sigma_{3}}(p, \varepsilon) \subset B_{\sigma_{1}}\left(p, \varepsilon_{1}\right) \cap B_{\sigma_{2}}\left(p, \varepsilon_{2}\right)$. Therefore, $B_{\sigma_{3}}(p, \varepsilon) \subset U_{p} \cap W_{p}$. Take $V_{p}=B_{\sigma_{3}}(p, \varepsilon)$. Then $V_{p}$ is a $\lambda_{\Omega}$-neighbourhood of $p$ such that $V_{p} \subset U_{p} \cap W_{p}$. Hence $\lambda_{\Omega}$ satisfies $N_{2}$.

(b) The proof is similar to that of (a).

Theorem 4.4. Let $(X, \Omega)$ be a generalized metric space and $\Omega$ satisfy the $\mathscr{V}$-property. Then every kernel in $(X, \Omega)$ is a perfect kernel.

Proof. Suppose that $\Omega_{0} \subset \Omega$ is a kernel in $(X, \Omega)$. Let $W_{1}, W_{2}, W_{3}, \ldots, W_{n} \in \lambda_{\Omega}$ with $W_{1} \cap W_{2} \cap \ldots \cap W_{n} \neq \emptyset$. By Theorem $4.3, W_{1} \cap W_{2} \cap$ $\ldots \cap \cap W_{n} \in \tilde{\lambda}_{\Omega}$. Since $\Omega_{0}$ is a kernel, there exists a metric $\sigma_{1} \in \Omega_{0}$ such that $i_{\sigma_{1}}\left(\cap_{i=1}^{n} W_{i}\right) \neq \emptyset$. Therefore, $\Omega_{0}$ is a perfect kernel in $(X, \Omega)$.

Theorem 4.5. Let $(X, \Omega)$ be a generalized metric space and $\Omega$ satisfy the $\mathscr{V}$-property. Then $(X, \Omega)$ is a weakly complete metric space if and only if $(X, \Omega)$ is a complete metric space.

Proof. Suppose $(X, \Omega)$ is a weakly complete metric space. Then there exists a kernel $\Omega_{0} \subset \Omega$ consisting of all complete metrics on $X$. By Theorem 4.4, $\Omega_{0}$ is a perfect kernel on $X$. Thus, there exists a perfect kernel $\Omega_{0} \subset \Omega$ consisting of all complete metrics on $X$. Therefore, $(X, \Omega)$ is a complete space. Since every complete metric space is a weakly complete metric space, the converse follows.

The following Theorem 4.6 gives the relations between $\lambda_{\Omega}$-open sets and neighbourhoods defined in a statistical metric space.

Theorem 4.6. Let $(S, F)$ be a statistical metric space. If the distribution function $F_{p q}\left(x_{i}\right)=1-\sigma_{i}(p, q)$ for $x_{i}>0, \sigma_{i} \in \Omega_{S}$ and $i \in \mathbb{N}$ where $\Omega_{S}$ is the collection of all metrics defined on $S$, then the following hold.

(a) Every $\tau$-neighbourhood on $S$ is a $\lambda_{\Omega_{S}}$-open set.

(b) Every $\tau_{e}$-neighbourhood on $S$ is a $\lambda_{\Omega_{S}}$-open set.

(c) Every $\tau_{R}$-neighbourhood on $S$ is a $\lambda_{\Omega_{S}}$-open set.

Proof. (a) Let $U$ be an arbitrary $\tau$-neighbourhood on $S$. Then $U=\left\{q \in S \mid F_{p q}\left(u_{1}\right)>1-v_{1}\right\}$ where $u_{1}, v_{1}>0$. By hypothesis, $U=\{q \in$ $\left.S \mid 1-\sigma_{1}(p, q)>1-v_{1}\right\}=\left\{q \in S \mid \sigma_{1}(p, q)<v_{1}\right\}=\left\{q \in S \mid q \in B_{\sigma_{1}}\left(p, v_{1}\right)\right\}$. Hence $U=B_{\sigma_{1}}\left(p, v_{1}\right)$ and so for each $x \in U$, there is a metric $\sigma \in \Omega_{S}$ and $\varepsilon>0$ such that $B_{\sigma}(x, \varepsilon) \subset U$. Therefore, $U \in \lambda_{\Omega_{S}}$. Hence every $\tau$-neighbourhood is a $\lambda_{\Omega_{S}}$-open set. 
(b) By Theorem 3.5, every $\tau_{e}$-neighbourhood on $S$ is a $\tau$-neighbourhood on $S$. Therefore, by (a), every $\tau_{e}$-neighbourhood on $S$ is a $\lambda_{\Omega_{S}}$-open set on $S$.

(c) Every $\tau_{R}$-neighbourhood on $S$ is a $\tau$-neighbourhood on $S$, by Theorem 3.7. By (a), every $\tau_{R}$-neighbourhood on $S$ is a $\lambda_{\Omega_{S}}$-open set on $S$.

Theorem 4.7. Let $(S, F)$ be a statistical metric space. If the distribution function $F_{p q}\left(x_{i}\right)=1-\sigma_{i}(p, q)$ for $x_{i}>0, \sigma_{i} \in \Omega_{S}$ and $i \in \mathbb{N}$, then the following hold.

(a) Every $\lambda_{\Omega_{S}}$-open set contains a $\tau$-neighbourhood on $S$.

(b) Every $\lambda_{\Omega_{S}}$-open set contains a $\tau_{e}$-neighbourhood on $S$.

Proof. We will present the detailed proof only for (b). Let $A \in \tilde{\lambda}_{\Omega_{S}}$ and $x \in A$. Then there is a metric $\sigma_{1} \in \Omega_{S}$ and $\varepsilon>0$ such that $B_{\sigma_{1}}(x, \varepsilon) \subset A$. Let $y \in B_{\sigma_{1}}(x, \varepsilon)$. Then $\sigma_{1}(x, y)<\varepsilon$ implies that $1-F_{x y}\left(u_{1}\right)<\varepsilon$ where $u_{1}>0$, by hypothesis. Take $f\left(u_{1}\right)=\varepsilon$. Then $F_{x y}\left(u_{1}\right)>1-f\left(u_{1}\right)$ and so $y \in\left\{z \in S \mid F_{x z}\left(u_{1}\right)>1-f\left(u_{1}\right)\right\}$. Take $U=\left\{z \in S \mid F_{x z}\left(u_{1}\right)>1-f\left(u_{1}\right)\right\}$. Then $U=\left\{z \in S \mid G_{x z}\left(u_{1}\right)<f\left(u_{1}\right)\right\}$ and $B_{\sigma_{1}}(x, \varepsilon) \subseteq U$. Since in a statistical metric space $G(p, q)=G_{p q}, U=\{z \in S \mid G(x, z)<f\}$. Therefore, $U$ is a $\tau_{e}$-neighbourhood on $S$. Let $t \in U$. Then $F_{x t}\left(u_{1}\right)>1-f\left(u_{1}\right)$ and so $1-\sigma_{1}(x, t)>1-f\left(u_{1}\right)$, by hypothesis. This implies that $\sigma_{1}(x, t)<f\left(u_{1}\right)$ which implies that $\sigma_{1}(x, t)<\varepsilon$, since $f\left(u_{1}\right)=\varepsilon$. Therefore, $t \in B_{\sigma_{1}}(x, \varepsilon)$. Hence $U=B_{\sigma_{1}}(x, \varepsilon)$. Thus, $U \subset A$. Hence $A$ contains a $\tau_{e}$-neighbourhood on $S$.

The following Theorem 4.8 shows that a collection of all $\lambda_{\Omega}$-open sets is a stack in statistical metric spaces.

Theorem 4.8. Let $(S, F)$ be a statistical metric space with a g-topology $v$. If the distribution function $F_{p q}\left(x_{i}\right)=1-\sigma_{i}(p, q)$ for $x_{i}>0, \sigma_{i} \in$ $\Omega_{S}, i \in \mathbb{N}$ where $v \in\left\{\tau, \tau_{e}\right\}$, then the following hold.

(a) The collection $\lambda_{\Omega_{S}}$ is a stack.

(b) If $W_{1}, W_{2}, \ldots, W_{n} \in \lambda_{\Omega_{S}}$ with $W_{1} \cap W_{2} \cap \ldots \cap W_{n} \neq \emptyset$, then $W_{1} \cap W_{2} \cap \ldots \cap W_{n} \in \lambda_{\Omega_{S}}$.

Proof. We will give a detailed proof only for $v=\tau$.

(a) Let $A \in \lambda_{\Omega_{S}}$ and $A \subset B$. By hypothesis and Theorem 4.7, $A$ contains a $\tau$-neighbourhood $W$ on $S$. This implies that $W \subset B$ which implies that $B \in \mathscr{N}(Z)$, since $\mathscr{N}(Z)$ is stack (Theorem 3.15). Therefore, $B \in \lambda_{\Omega_{S}}$, by hypothesis and Theorem 4.6. Hence $\lambda_{\Omega_{S}}$ is a stack.

(b) Let $V_{1}, V_{2}, \ldots, V_{n} \in \lambda_{\Omega_{S}}$ with $V_{1} \cap V_{2} \cap \ldots \cap V_{n} \neq \emptyset$. Choose $x \in V_{1} \cap V_{2} \cap \ldots \cap V_{n}$. Then there exist $\sigma_{i} \in \Omega_{S}, \varepsilon_{i}>0$ such that $B_{\sigma_{i}}\left(x, \varepsilon_{i}\right) \subset V_{i}$ for $i=1$ to $n$ and so $\cap_{i=1}^{n} B_{\sigma_{i}}\left(x, \varepsilon_{i}\right) \subset \cap_{i=1}^{n} V_{i}$. As in the proof of Theorem 4.7, we get that $B_{i}=W_{i}$ where $B_{i}=B_{\sigma_{i}}\left(x, \varepsilon_{i}\right)$ and $W_{i}$ is a $\tau$-neighbourhood on $S$ for $i=1$ to $n$. Therefore, $\cap_{i=1}^{n} W_{i} \subset \cap_{i=1}^{n} V_{i}$. By Theorem 3.18, $\cap_{i=1}^{n} W_{i}$ is a $\tau$-neighbourhood on $S$. Thus, $\cap_{i=1}^{n} W_{i}$ is a $\lambda_{\Omega_{S}}$-open set, by Theorem 4.6 and hence $\cap_{i=1}^{n} V_{i} \in \lambda_{\Omega_{S}}$, by (a).

Theorem 4.9. Let $(S, F)$ be a statistical metric space with a g-topology $\tau$ or $\tau_{e}$. If the distribution function $F_{p q}\left(x_{i}\right)=1-\sigma_{i}(p, q)$ for $x_{i}>0, \sigma_{i} \in \Omega_{S}, i \in \mathbb{N}$ and if $\Omega_{0} \subset \Omega_{S}$ is a kernel in $\left(S, \Omega_{S}\right)$, then it is a perfect kernel in $\left(S, \Omega_{S}\right)$.

Proof. Let $(S, F)$ be a statistical metric space with $\tau$. Suppose $\Omega_{0} \subset \Omega_{S}$ is a kernel in $\left(S, \Omega_{S}\right)$. Let $V_{1}, V_{2}, V_{3}, \ldots, V_{n} \in \lambda_{\Omega_{S}}$ with $V_{1} \cap V_{2} \cap$ $\ldots \cap V_{n} \neq \emptyset$. By hypothesis and Theorem 4.8, $V_{1} \cap V_{2} \cap \ldots \cap V_{n} \in \tilde{\lambda}_{\Omega_{S}}$. Since $\Omega_{0}$ is a kernel, there exists a metric $\sigma_{1} \in \Omega_{0}$ such that $i_{\sigma_{1}}\left(\cap_{i=1}^{n} V_{i}\right) \neq \emptyset$. Therefore, $\Omega_{0}$ is a perfect kernel in $\left(S, \Omega_{S}\right)$.

Let $(S, F)$ be a statistical metric space with $\tau_{e}$. By the same argument as in above and Theorem 4.8, we can prove that $\Omega_{0}$ is a perfect kernel in $\left(S, \Omega_{S}\right)$

Theorem 4.10. Let $(S, F)$ be a statistical metric space with a g-topology $\tau$ or $\tau_{e}$. If the distribution function $F_{p q}\left(x_{i}\right)=1-\sigma_{i}(p, q)$ for $x_{i}>0, \sigma_{i} \in \Omega_{S}, i \in \mathbb{N}$ and if $\left(S, \Omega_{S}\right)$ is a weakly complete metric space, then $\left(S, \Omega_{S}\right)$ is a complete metric space.

Proof. Let $(S, F)$ be a statistical metric space with $\tau$. Suppose $\left(S, \Omega_{S}\right)$ is a weakly complete space. Then there exists a kernel $\Omega_{0} \subset \Omega_{S}$ consisting of all complete metrics on $S$. By hypothesis and Theorem 4.9, $\Omega_{0}$ is a perfect kernel on $S$. Thus, there exists a perfect kernel $\Omega_{0} \subset \Omega_{S}$ consisting of all complete metrics on $S$. Therefore, $\left(S, \Omega_{S}\right)$ is a complete metric space.

Suppose that $(S, F)$ is a statistical metric space with $\tau_{e}$. By the same argument as in above and Theorem 4.9 , we can prove that $\left(S, \Omega_{S}\right)$ is a complete metric space.

\section{Conclusion}

This article provide the basis for carrying out analysis in statistical metric spaces, in particular for the development of various $g$-topologies, neighbourhoods defined in a statistical metric space and also the improvement of $\lambda_{\Omega}$-open sets in a generalized metric space. We have given more examples of the neighbourhoods defined in a statistical metric space and the special kind of relationship between various $g$-topologies defined by Thorp in a SM space. Also, new properties for $\lambda_{\Omega}$-open sets in a generalized metric space have presented. We have given partial answer to the following questions raised by Thorp in statistical metric spaces:

What are the necessary and sufficient conditions that the $g$-topology of typeV to be of typeV $V_{D}$ ?

What are the necessary and sufficient conditions that the $g$-topology of $t y p e V_{\alpha}$ to be the $g$-topology of $t y p e V_{D}$ ?

What conditions are both necessary and sufficient for the $g$-topology of type $V_{\alpha}$ to be a topology? 


\section{Acknowledgment}

The authors would like to thank the referees for their deep observations and valuable comments to improve this article.

\section{References}

[1] M. Fréchet, Sur quelques points du calcul fonctionnel, Rend. Circ. Mat. Palermo, 22 (1906), 1 - 74.

[2] E. Korczak-Kubiak, A. Loranty and R. J. Pawlak, Baire Generalized topological spaces, Generalized metric spaces and infinite Games, Acta Math. Hungar., 140 (2013), 203 - 231.

[3] K. Menger, statistical metrics, Proc. Nat. Acad. of Sci., U.S.A. 28 (1942), 535 - 537.

[4] K. Menger, Probabilistic theories of relations, Ibid., 37 (1951), 178 - 180.

[5] K. Menger, Probabilistic geometry, Ibid., 37 (1951), 226 - 229.

[6] W. K. Min, On weak neighborhood systems and spaces, Acta Math. Hungar., 121 (3) (2008), 283 - 292.

[7] B. Schweizer, A. Sklar and B. Thorp, The metrization of statistical metric spaces, Pacific J. Math., 10 (2) (1960), 673 - 675.

[8] E. O. Thorp, Best possible triangle inequalities for statistical metric spaces, Proc. Amer. Math. Soc., 11 (1960), 734 - 740.

[9] E. O. Thorp, Generalized topologies for statistical metric spaces, Fund. Math., Li, 51 (1962), 9 - 21.

[10] A. Wald, On a statistical generalization of metric spaces, Proc. Natl. Acad. Sci. USA., 29 (1943), 196 - 197. 